\title{
Photometric distances to six bright resolved galaxies ${ }^{\star}$
}

\author{
I.O. Drozdovsky ${ }^{1}$ and I.D. Karachentsev ${ }^{2}$ \\ 1 Astronomical Institute, St.-Petersburg State University, Petrodvoretz 198904, Russia \\ 2 Special Astrophysical Observatory, N. Arkhyz, KChR 357147, Russia
}

Received September 27; accepted October 18, 1999

\begin{abstract}
We present photometry of the brightest stars in six nearby spiral and irregular galaxies with corrected radial velocities from 340 to $460 \mathrm{~km} \mathrm{~s}^{-1}$. Three of them are resolved into stars for the first time. Based on luminosity of the brightest blue stars we estimate the following distances to the galaxies: 5.0 Mpc for NGC 784, 9.2 Mpc for NGC 2683, 8.9 Mpc for NGC 2903, 4.1 Mpc for NGC 5204, 6.8 Mpc for NGC 5474, and 8.7 Mpc for NGC 5585.
\end{abstract}

Key words: galaxies: distances — galaxies: general

\section{Introduction}

This paper completes a series of publications devoted to photometry of the brightest stars in nearby late type galaxies having radial velocities $V_{0}<500 \mathrm{~km} \mathrm{~s}^{-1}$ from the sample by Kraan-Korteweg \& Tammann (1979) with the aim to measure their distances regardless of velocities. Distance moduli derived via the luminosity of blue and red supergiants (Sandage \& Tammann 1974; de Vaucouleurs 1979) have a typical error of $0.4-0.5$, which exceeds the mean error of $0 \cdot 1-0.2$ moduli derived from the luminosity of cepheids or the tip of red giant branch. However when applied to the dwarf galaxies consisting of about $90 \%$ of the Local Volume population, the method of the brightest stars gives a $2-3$ times better accuracy than the widespread method of Tully-Fisher (1977). The initial sample of 179 galaxies in the Local Volume with $V_{0}<500 \mathrm{~km} \mathrm{~s}^{-1}$ (Kraan-Korteweg \& Tammann 1979) was increased by Karachentsev (1994) to as many as 216 galaxies. Among them almost all 120 northern galaxies were imaged with a CCD at the $6-\mathrm{m}$ SAO telescope and the 2.5-m Nordic telescope in La Palma (Karachentsev

Send offprint requests to: I. Drozdovsky;

e-mail: dio@astro.spbu.ru

* Data available in electronic form at the CDS via anonymous ftp to cdsarc.u-strasbg.fr (130.79.128.5) or via http://cdsweb.u-strasbg.fr/Abstract.html et al. 1994; Georgiev et al. 1997; Makarova et al. 1997; Karachentsev et al. 1997; Makarova \& Karachentsev 1998; Karachentsev \& Drozdovsky 1998; Tikhonov \& Karachentsev 1998). Besides several early type objects (NGC 404, NGC 4150, NGC 4736, NGC 4826, and Maffei 1) all of the galaxies are resolved into stars, moreover most of them for the first time.

Here we present large-scale images of three previously unobserved galaxies: NGC 784, NGC 2683, NGC 2903, as well as of three galaxies, NGC 5204, NGC 5474, and NGC 5585, from the M 101 group imaged with a higher angular resolution.

\section{Observations and photometry}

The first four galaxies were observed in February 1995 at the 2.5-m Nordic telescope with a subarcsec seeing. A CCD chip of $1 \mathrm{~K} \times 1 \mathrm{~K}$ pixels provided a field of view of $3^{\prime} \times 3^{\prime}$ with a resolution of $0^{\prime \prime} 176 /$ pixel. The last two galaxies were observed on March-June 1997 at the prime focus of the $6-\mathrm{m}$ telescope where a CCD camera provided a field of $2.3 \times 2$ '. 3 with a resolution of $0{ }^{\prime \prime} \cdot 137 /$ pixel. The average seeing was about $1^{\prime \prime} 5$. The exposure times in the $B, V$, $R, I$ filters of Johnson-Cousins system, as well the seeings (FWHM) are given in Table 1. Equatorial standards from Landolt (1992) were used for the frame calibration.

Photometry of stellar objects in these galaxies was carried out with the DAOPHOT/ALLSTAR package (Stetson 1987). In some cases of crowded stellar field with high brightness gradients we repeated photometry after subtracting their frames smoothed with a median filter.

\section{Distance estimates for the galaxies}

$$
\begin{aligned}
& \text { 3.1. } N G C 784=U G C 1501=P G C 7671=I R A S \\
& 01582+2836
\end{aligned}
$$

This irregular galaxy seen nearly edge-on with angular dimensions of $6 ! 6 \times 1 ! 5$ and an integral apparent magnitude 
Table 1. Journal of observations

\begin{tabular}{|c|c|c|c|c|}
\hline NGC & Date & Filter & Exp.(s) & Seeing \\
\hline \multirow[t]{2}{*}{784} & 1995, Feb. 7 & $\bar{V}$ & 300 & $0^{\prime \prime} 6$ \\
\hline & & $I$ & 300 & \\
\hline \multirow[t]{3}{*}{$2683 \mathrm{~s}$} & 1995, Feb. 4 & $B$ & 900 & $0 . .8$ \\
\hline & & $V$ & 600 & \\
\hline & & $I$ & 600 & \\
\hline \multirow[t]{3}{*}{$2683 n$} & 1995, Feb. 4 & $B$ & 900 & $0 . .8$ \\
\hline & & $V$ & 600 & \\
\hline & & $I$ & 600 & \\
\hline \multirow[t]{2}{*}{$2903 \mathrm{~s}$} & 1995, Feb. 4 & $V$ & 600 & $0^{\prime \prime} 6$ \\
\hline & & $I$ & 600 & \\
\hline \multirow[t]{2}{*}{$2903 n$} & 1995, Feb. 4 & $V$ & 600 & $0 . .6$ \\
\hline & & $I$ & 600 & \\
\hline \multirow[t]{2}{*}{5204} & 1995, Feb. 7 & $B$ & 200 & $0 . .5$ \\
\hline & & $V$ & 100 & \\
\hline \multirow[t]{3}{*}{$5474 \mathrm{c}$} & 1997, June 7 & $\bar{V}$ & 600 & $1^{\prime \prime} .5$ \\
\hline & & $R$ & 600 & \\
\hline & & $I$ & 600 & \\
\hline \multirow[t]{2}{*}{$5474 \mathrm{~s}$} & 1997, June 10 & $V$ & 600 & $1^{\prime \prime} 3$ \\
\hline & & $I$ & 600 & \\
\hline \multirow[t]{5}{*}{5585} & 1997, Mar. 11 & $V$ & 600 & $1^{\prime \prime} 7$ \\
\hline & & $R$ & 600 & \\
\hline & & $I$ & 600 & \\
\hline & 1997, June 11 & $V$ & 600 & $1^{\prime \prime} .5$ \\
\hline & & $I$ & 600 & \\
\hline
\end{tabular}

of $B_{\mathrm{T}}=12.23$ has a radial velocity of $V_{\mathrm{h}}=198 \mathrm{~km} \mathrm{~s}^{-1}$ (NASA Extragalactic Database $=$ NED). In spite of its expected small distance, NGC 784 has not yet been resolved into stars. Broeils \& van Woerden (1994) studied the structure and kinematics of NGC 784 in the Hi line, showing that its neutral hydrogen content as well as rotational amplitude are typical of an irregular galaxy. The central part of NGC 784 is represented in Fig. 1 from our $V$ CCD frame. Among star-like objects found by DAOPHOT in this field we put into a Colour-Magnitude Diagram (=CMD) 84 stars, whose image parameters satisfy the conditions $|\mathrm{SHARP}|<2,|\mathrm{CHI}|<2$, and $\sigma(m)<0.2$. The CMD for them, Fig. 2, shows the presence of some blue stars. Following Sandage \& Tammann (1974) and de Vaucouleurs (1979) we estimated the distance modulus via the relation

$(m-M)_{0}=1.51 \cdot\langle B(3 B)\rangle-0.51 \cdot B_{\mathrm{T}}-A_{B}+4.14$

between the mean apparent magnitude of three blue supergiant candidates, $\langle B(3 B)\rangle$, and the integral magnitude of the galaxy, $B_{\mathrm{T}}$, where $A_{B}$ is the galactic extinction. The numerical coefficients were derived by Karachentsev \& Tikhonov (1994) from galaxies with distances known via cepheids. In the cases where a galaxy was observed in the $V$ and $I$ bands only, the $(B-V)$ color was derived from the relation $(B-V)=0.83 \cdot(V-I)$ defined by the standard Landolt's stars with $V-I<1$. With $\langle B(3 B)\rangle=20.39$ and $A_{B}=0.22$ we derive for NGC 784 a distance modulus $(m-M)_{0}=28.47$ or $D=5.0 \mathrm{Mpc}$.

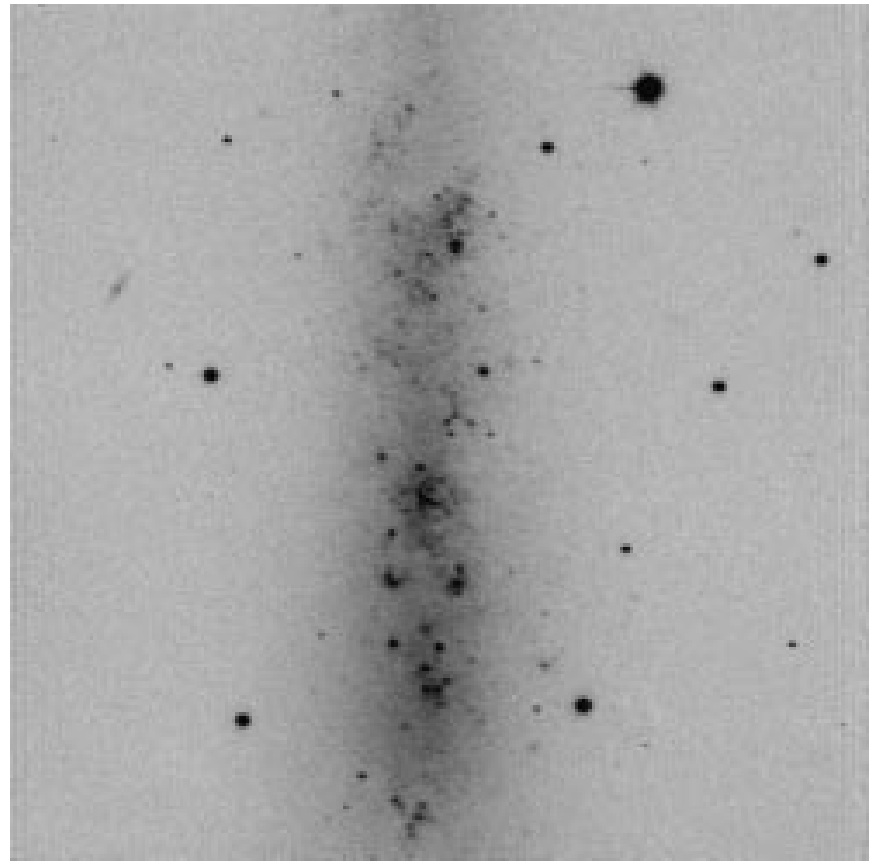

Fig. 1. NGC 784 in the $V$ band. As for all the other images, North is to the top, East is to the left. The upper right corner corresponds to $X=0, Y=0$ of the frame coordinates given in the photometry tables, i.e. $X$ increases to the East and $Y$ to the South

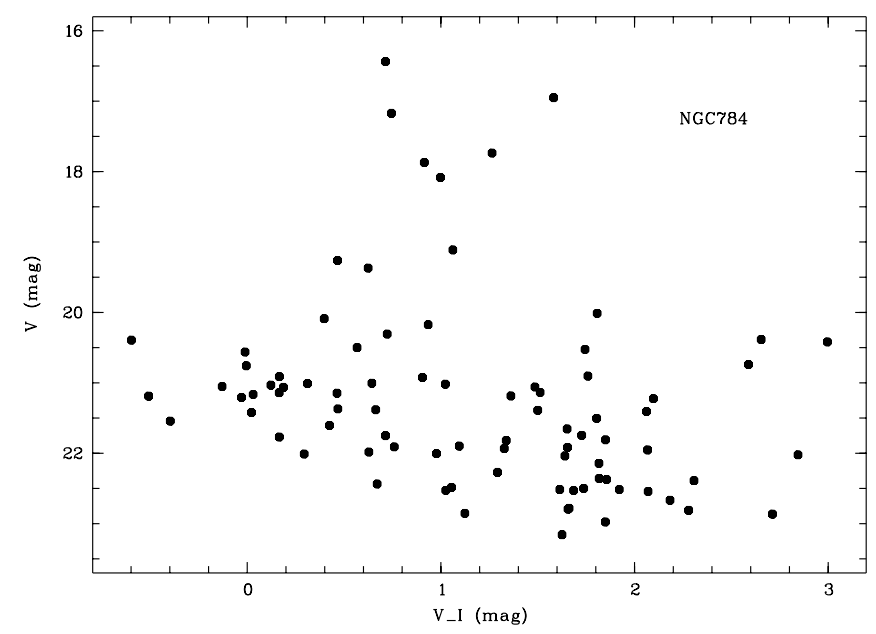

Fig. 2. Colour-Magnitude diagram for 84 stars in NGC 784

The basic parameters of NGC 784 and of the other considered galaxies are given in Table 2. Its first six lines indicate the standard major and minor diameters of the galaxy, its integral apparent magnitude, galactic extinction, morphological type, and heliocentric radial velocity from the NED. The next lines contain the radial velocity with respect to the centroid of the Local Group (Karachentsev \& Makarov 1996), number of measured brightest stars, mean magnitude and colour of three 
brightest blue stars, the distance modulus and linear distance of the galaxy, and its absolute blue magnitude.

Note that recent Hi observations by Huchtmeier et al. (2000) reveal in the NGC 784 neighbourhood two dwarf galaxies, kk16 and kk17, with radial velocities +399 and $+348 \mathrm{~km} \mathrm{~s}^{-1}$, respectively, which are probable companions of NGC 784 .

3.2. $N G C 2683=U G C 4641=P G C 24930=I R A S$ $08493+3336$

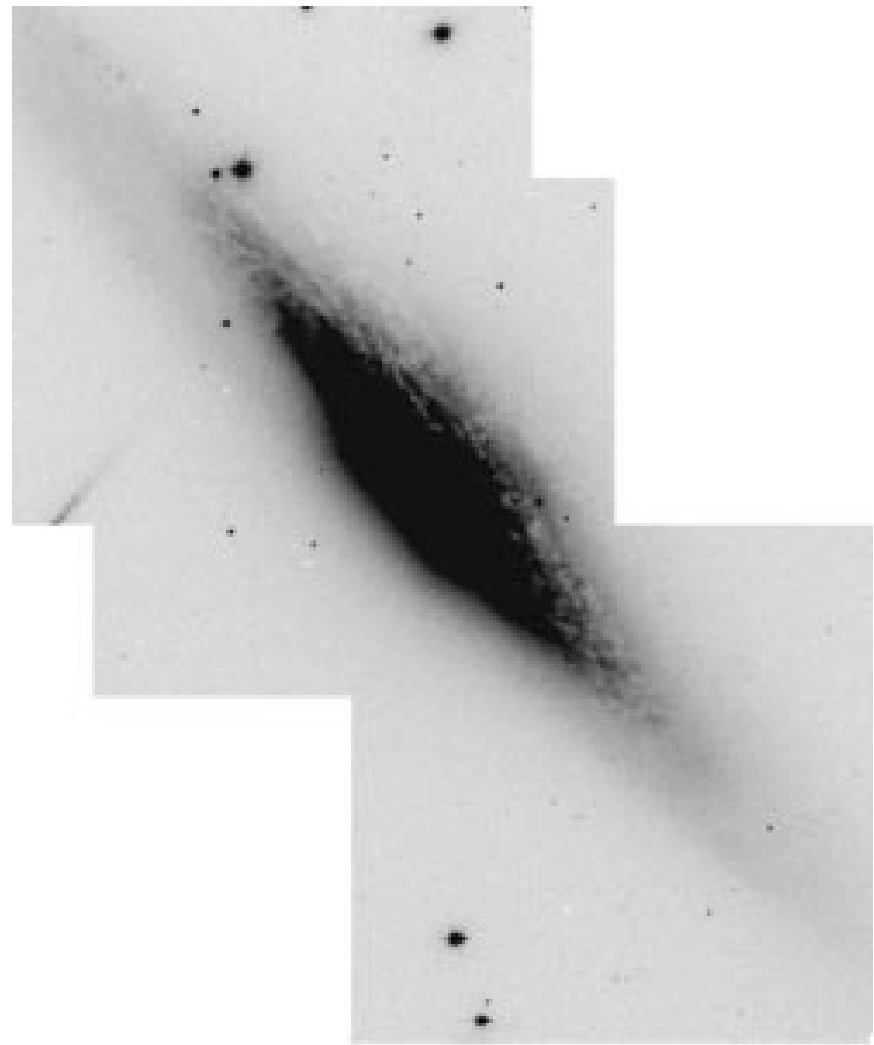

Fig. 3. Mosaic NGC 2683 image in the $R$ band

A view of this Sb-type galaxy is shown in a mosaic (Fig. 3) constructed from three frames in the $R$ filter with $30 \mathrm{~s}$ exposure each. Figure 4 presents images of the northern (a) and southern (b) parts of the galaxy in the $V$ band after subtracting the frames smoothed with a median filter with a $10 \times$ FWHM window. As one can see from these images, the whole body of the galaxy is spotted with dusty lines, which impedes accurate photometry of stars, especially in the $B$ band. The results of our photometry of 327 stars and star-like objects are presented in the diagram $V \propto V-I$ (Fig. 5). The stars from the northern galaxy side are indicated by dark circles, and the southern part stars are marked by open boxes. A considerable part of the brightest blue objects have a diffuse or elongated shape. After removing them we selected three
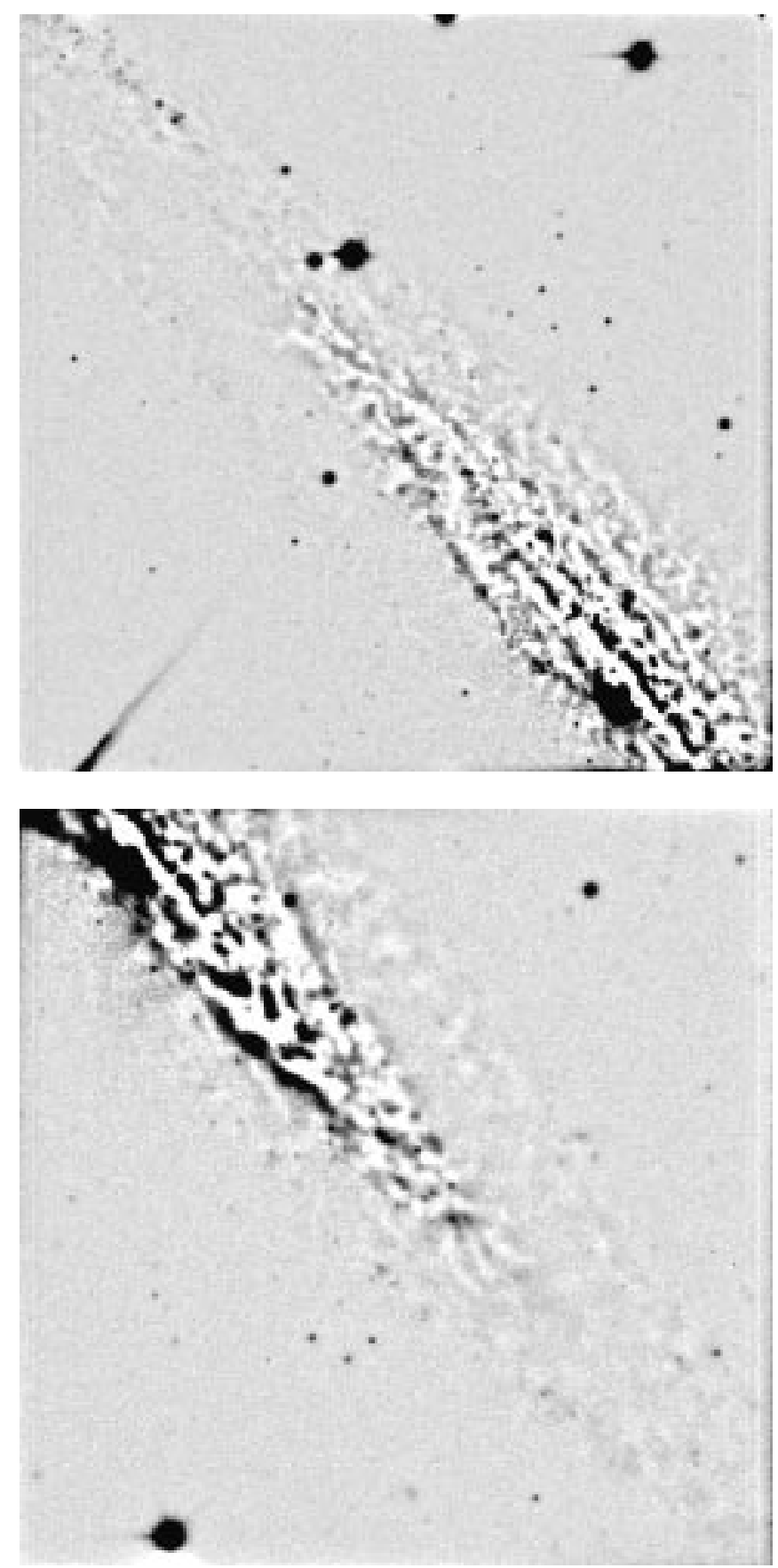

Fig. 4. Northern (top) and southern (bottom) parts of NGC 2683 in $V$ band

stars as blue supergiant candidates. Their mean apparent magnitude, 20.64, yields a distance modulus of 29.81 with $A_{B}=0.07$. Note that the the brightest red $(B-V>1.6)$ stars in the galaxy have $V=22.0$, which corresponds to a distance modulus of about 30.0 that agrees with the "blue" modulus. According to Tully (1988) NGC 2683 is situated in the scattered cloud "Leo spur", which probably moves with a peculiar velocity of about $-200 \mathrm{~km} \mathrm{~s}^{-1}$ with respect to the Local "pancake". This can explain the 


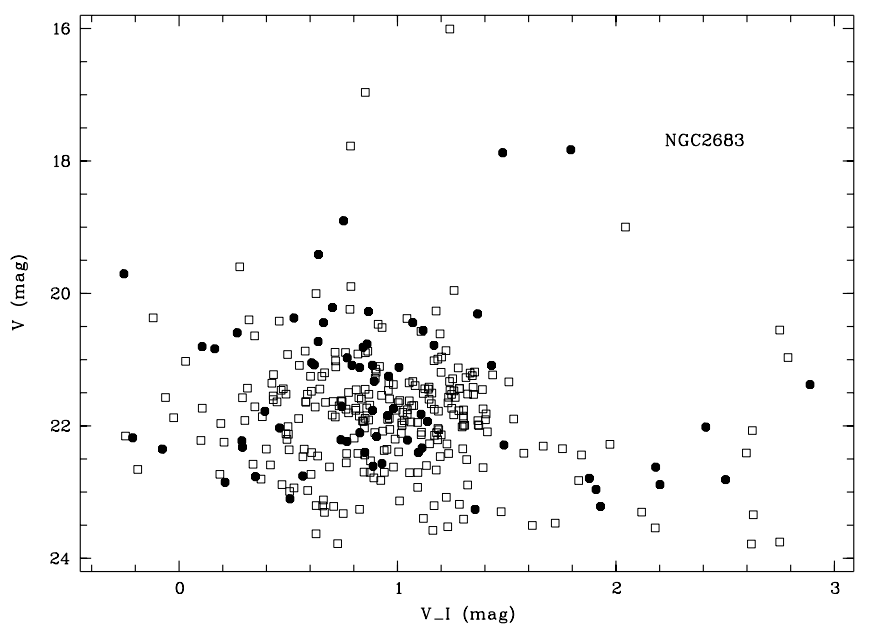

Fig. 5. CMD for 327 stars in NGC 2683. The filled circles are for stars measured in the northern region, the open squares indicate stars in the southern side

low radial velocity of the galaxy, $V_{0}=364 \mathrm{~km} \mathrm{~s}^{-1}$, at its rather large distance of $9.2 \mathrm{Mpc}$. From Karachentseva \& Karachentsev (1998) and Huchtmeier et al. (2000) there are two dwarf companions in the vicinity of NGC 2683: kk69 with $V_{\mathrm{h}}=420 \mathrm{~km} \mathrm{~s}^{-1}$ and the dwarf spheroidal system kk70. The rotation curve for NGC 2683 was studied in the HI line by Broeils \& van Woerden (1994).

\section{3. $N G C 2903=U G C 5079=K I G 347=P G C 27077$ $=\operatorname{IRAS} 09292+2143$}

This large $\left(12 ! 6 \times 6{ }^{\prime} 0\right)$ bright $\left(B_{\mathrm{T}}=9.68\right)$ spiral galaxy similar to M 81 still remained unresolved into stars. In its wide vicinity there is no other bright galaxy. This is why Karachentseva (1973) included NGC 2903 in her Catalog of isolated galaxies (KIG). Within $3^{\circ}$ around NGC 2903 there are two dwarf galaxies: UGC 5086 and F565-v1 imaged by Makarova \& Karachentsev (1998). An image of NGC 2903 from the Digital Sky Survey is shown in Fig. 6. Our two CCD frames indicated by the boxes cover the basic part of its spiral pattern except the faint outer arms. Reproductions of the northern and southern CCD images are presented in Fig. 7 after subtracting frames smoothed with a median filter of $10 \times$ FWHM window. The results of the photometry of 273 brightest stars are shown in $V \propto V-I$ diagram (Fig. 8). As in the case of NGC 2683, slightly diffuse objects prevail amongst the brightest blue ones. They are probably compact HII regions and multiple stars. As blue supergiant candidates, we pick three stars. Their mean apparent magnitude, 20.27, yields with galactic extinction of 0.07 a distance modulus of 29.74 or $D=8.9 \mathrm{Mpc}$. Being located in the same Leo spur cloud as NGC 2683, the spiral galaxy NGC 2903 has also a low radial-velocity to distance ratio: $H=V_{0} / D=$ $50 \mathrm{~km} \mathrm{~s}^{-1} / \mathrm{Mpc}$ (line 14 in Table 2).

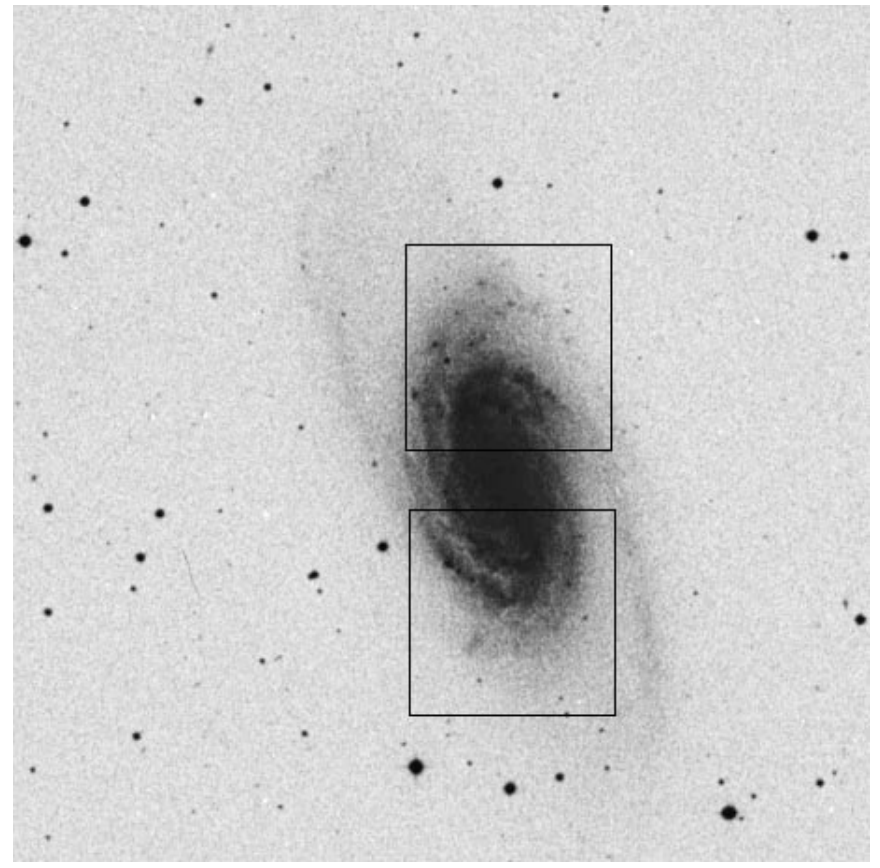

Fig. 6. Digital Sky Survey image of NGC 2903. The squares indicate the position of our frames

\section{4. $N G C 5204=U G C 8490=P G C 47368=I R A S$ $13274+5840$}

A complete image of this irregular galaxy in the $B+V$ bands is shown in Fig. 9 after subtraction of the median smoothed frame. It shows many more resolved stars than the reproduction in the Atlas of Sandage \& Bedke (1988). Despite the short exposures (100 and 200 s), photometry of our frames reveals 131 stars whose CMD is presented in Fig. 10. For three brightest blue stars we derived $\langle B(3 B)\rangle=19.81$ which is in good agreement with the previous estimate by Karachentsev et al. (1994) 19.92 . According to this paper, NGC 5204 is located in the outskirts of the M 101 group. However, its distance, $4.1 \mathrm{Mpc}$, seems to be considerably lower than the average distance of other members of the group, 6.9 Mpc.

\section{5. $N G C 5474=U G C 9013=V V 344=\operatorname{Arp} 26=$ $P G C 50216=I R A S 14031+5354$}

The structure of this nearest companion to M 101 is strongly disturbed by tidal interaction. A view of the galaxy imaged in the $R$ band is shown in Fig. 11. It has been taken from the archive of the Isaac Newton telescope (La Palma). Positions of two CCD frames obtained with the $6-\mathrm{m}$ telescope are indicated by boxes. The reproduction of the southern image in the $I$ band is presented in Fig. 12. The results of photometry of 298 brightest stars in both fields are given in the diagram $V \propto V-I$ (Fig. 13). For three brightest blue supergiant candidates we derived the mean apparent magnitude $\langle B(3 B)\rangle=20.37$, 

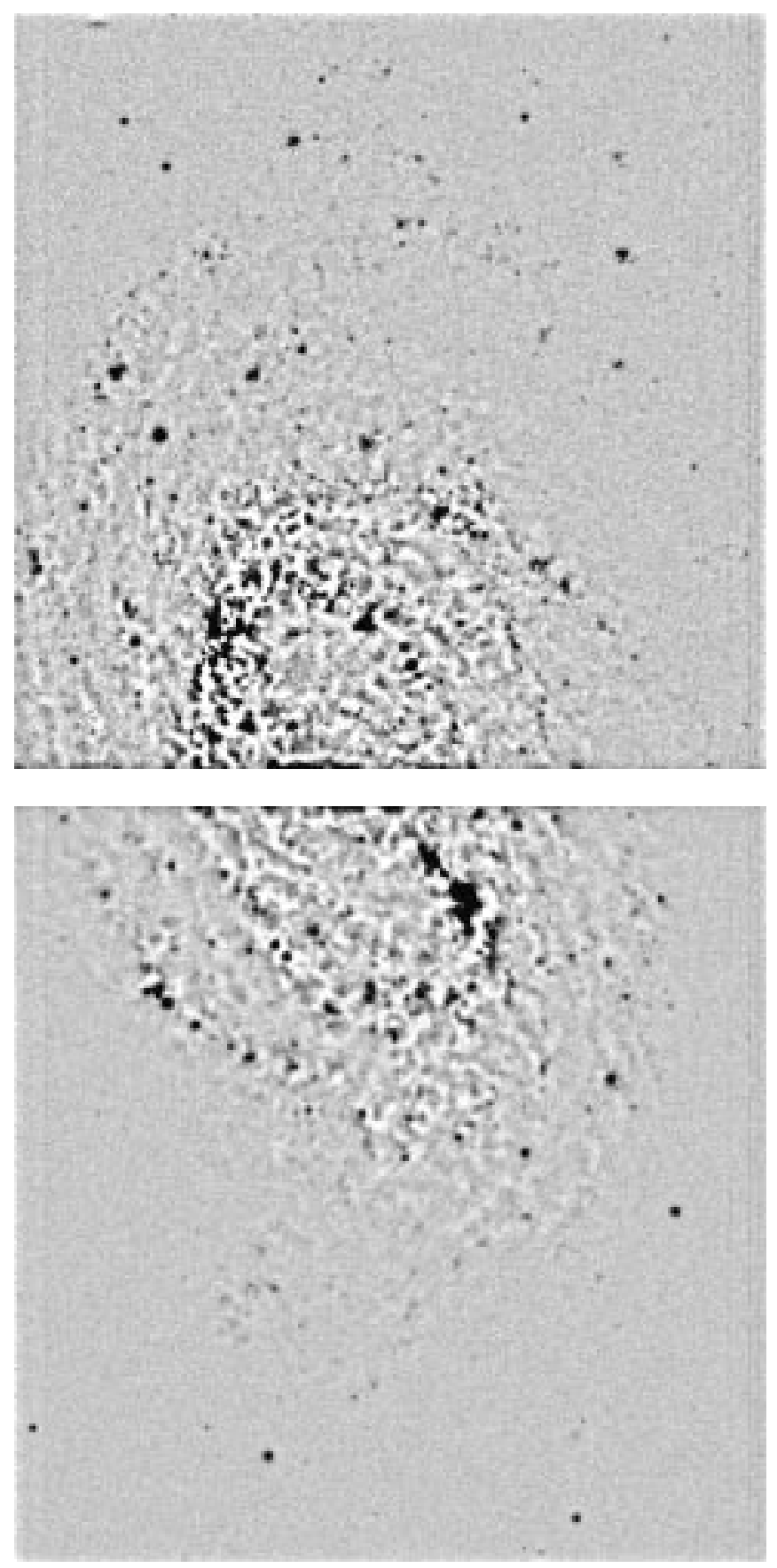

Fig. 7. Northern (top) and southern (bottom) parts of NGC 2903 in the $V$ band

which coincides with the previous estimate, 20.36, made by Karachentsev et al. (1994). The distance modulus of NGC 5474, $(m-M)_{0}=29.15$, agrees well with the modulus of M 101 (29.26) measured via cepheids (Alves \& Cook 1995).

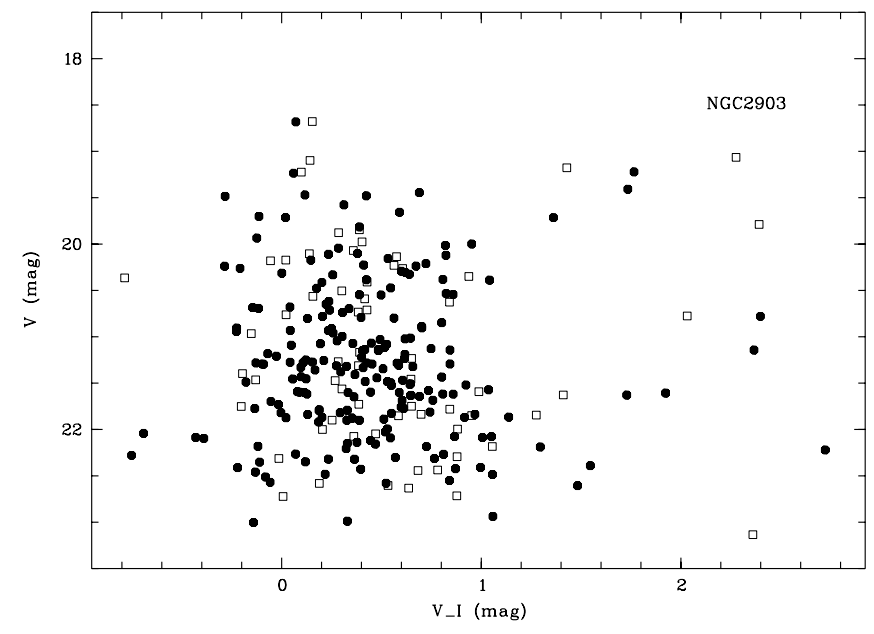

Fig. 8. CMD for 273 brightest stars in NGC 2903. The filled circles correspond to stars measured in the northern region and open squares indicate stars in the southern side

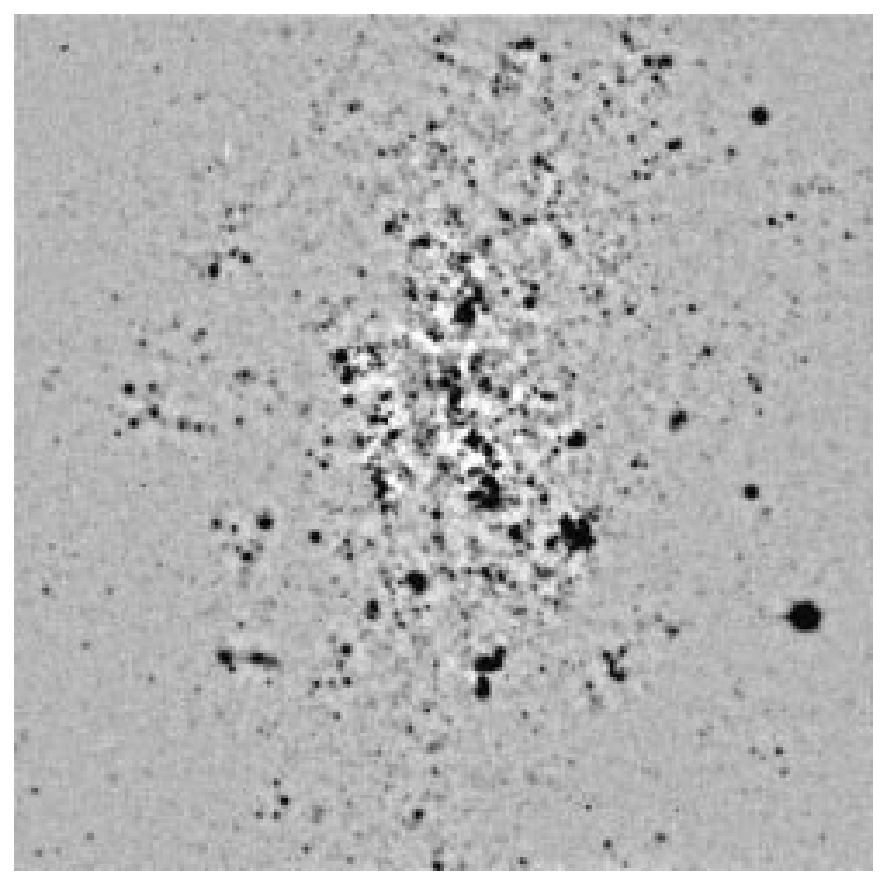

Fig. 9. Composite $B+V$ image of the central part of NGC 5204 after subtraction of the median smoothed frame

\section{6. $N G C 5585=U G C 9179=K I G 624=P G C 51210$ $=\operatorname{IRAS} 14181+5657$}

As the two previous galaxies, NGC 5585 is a probable member of the M 101 group, but it looks rather isolated with respect to the neighbouring galaxies. Fig. 14 reproduces a view of NGC 5585 in the $I$ band taken from the archive of the John Kapteyn telescope (La Palma).

Figure 15 shows the main body of the galaxy from the CCD image obtained with the 6-m telescope in the $I$ band after subtraction of the median smoothed frame. 


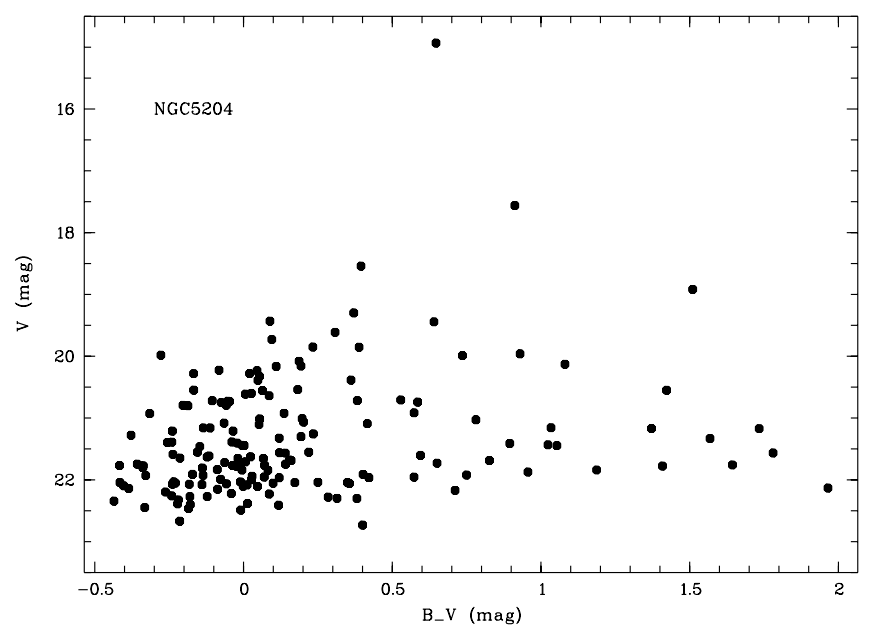

Fig. 10. $V$ vs. $(B-V) \mathrm{CM}$ diagram of the 131 stars in the area of NGC 5204

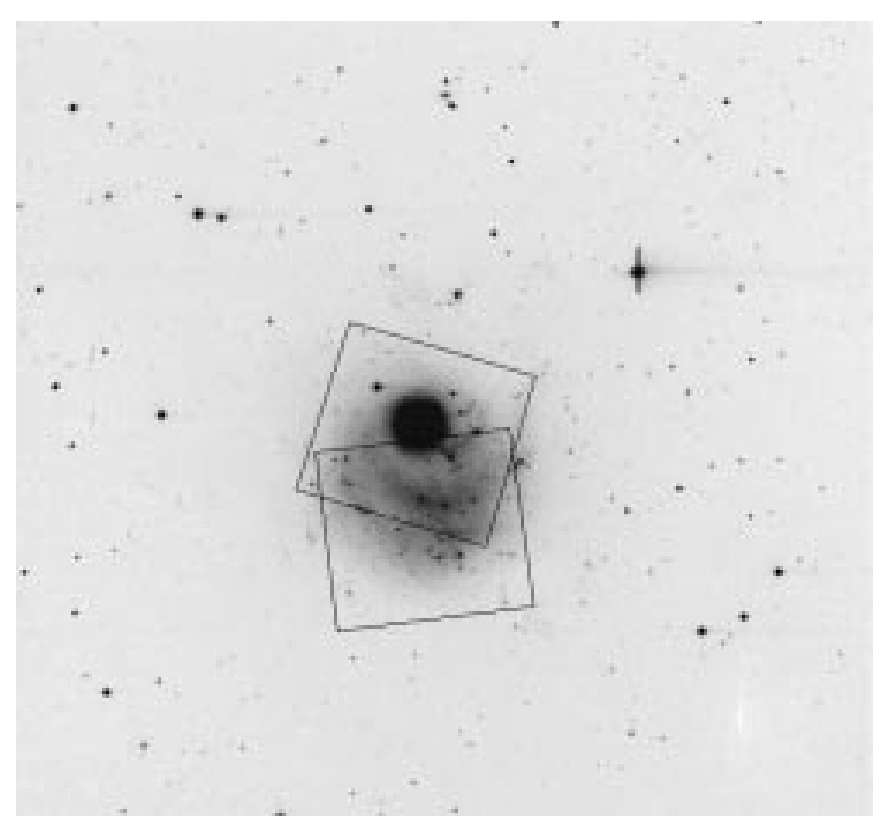

Fig. 11. $R$ band image of NGC 5474 from the Isaac Newton Telescope Archive. The two squares correspond to $6 \mathrm{~m}$ BTA CCD frames

The results of stellar photometry averaged over two observing runs are presented as a CMD, Fig. 16. The mean apparent magnitude of the three brightest blue stars is $\langle B(3 B)\rangle=20.71$, which agrees better with the old estimate of Sandage \& Tammann (1974) 20.9, than with the value of 20.02 derived by Karachentsev et al. (1994). However, all three brightest blue stars used by Karachentsev et al. (1994) are situated on the NE side of NGC 5585 beyond the boundaries of our present frame. Despite the somewhat uncertain distance of NGC 5585, 5.7 Mpc (former estimate), and 8.7 Mpc (the present paper), this galaxy seems to be peripheric member of the M 101 group.
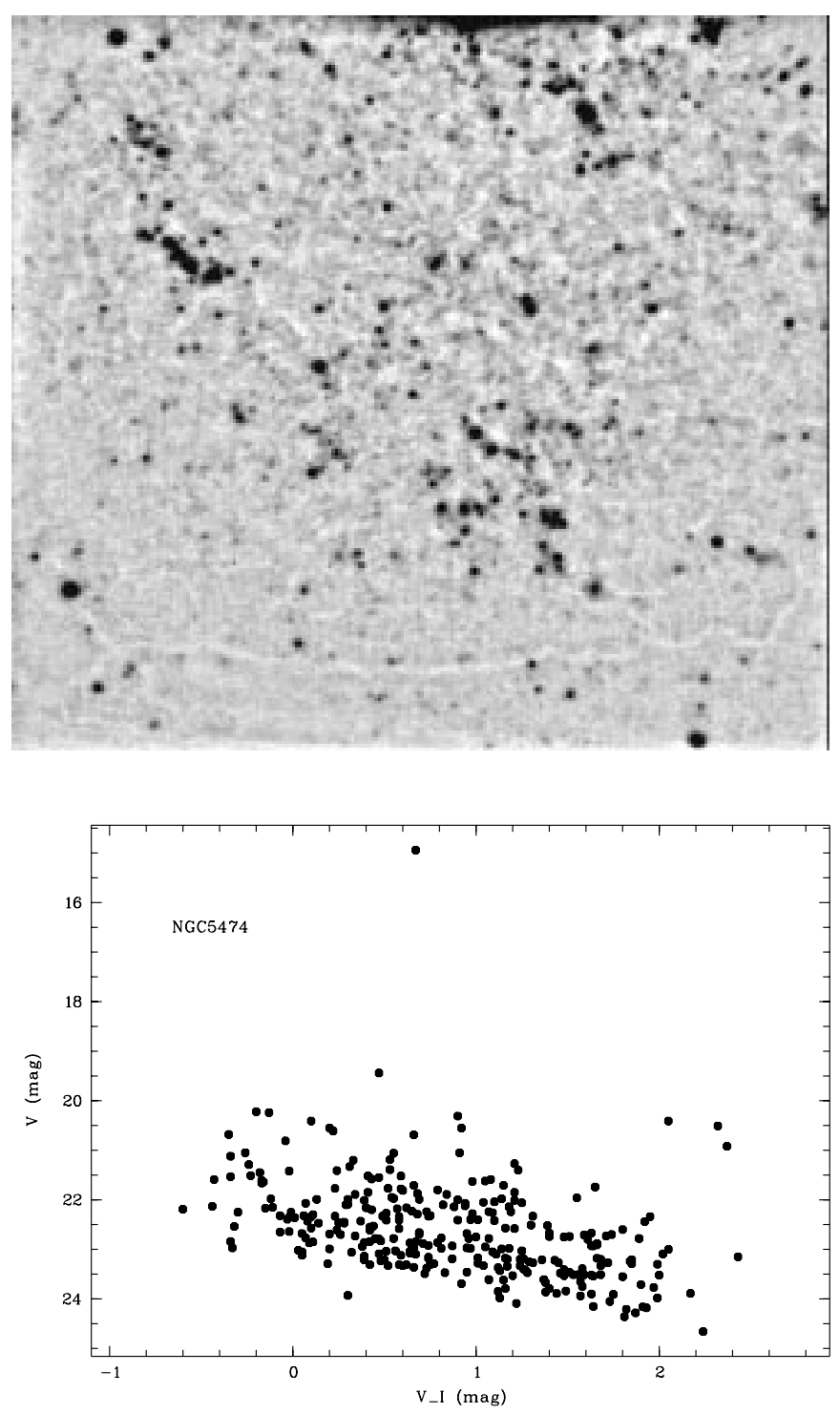

Fig. 13. Colour-magnitude diagram for 298 stars in NGC 5474

\section{Summary}

We obtained large scale CCD images in the $B, V, R$ and $I$ bands for six spiral and irregular galaxies with corrected radial velocities in the range of $340-460 \mathrm{~km} \mathrm{~s}^{-1}$. All the galaxies are well resolved into stars. Three of them have been resolved for the first time. Based on the luminosity of the brightest blue stars we determine their distances, which are collected in Table 2 together with other integral parameters of the galaxies. All the galaxies are known IRAS sources. Except NGC 5474, the galaxies seem well isolated systems, whose crossing time with respect to their neighbours is comparable with the cosmic expanding time, $1 / H$. Individual estimates of the Hubble parameter, $H=V_{0} / D$, lie in the range of $40-83 \mathrm{~km} \mathrm{~s}^{-1} / \mathrm{Mpc}$, showing the tendency for a decrease in $H$ with 
Table 2. Basic parameters of the resolved galaxies

\begin{tabular}{|lcccccc|}
\hline \multicolumn{1}{|c}{ Parameter } & $\mathrm{N} 784$ & $\mathrm{~N} \mathrm{2683}$ & $\mathrm{N} \mathrm{2903}$ & $\mathrm{N} \mathrm{5204}$ & $\mathrm{N} \mathrm{5474}$ & $\mathrm{N} \mathrm{5585}$ \\
\hline$a_{2} 5,\left({ }^{\prime}\right)$ & 6.6 & 9.3 & 12.6 & 5.0 & 4.8 & 5.8 \\
$b_{2} 5,\left({ }^{\prime}\right)$ & 1.5 & 2.2 & 6.0 & 3.0 & 4.3 & 3.7 \\
$B_{\mathrm{T}},(\mathrm{mag})$ & 12.23 & 10.64 & 9.68 & 11.73 & 11.28 & 11.20 \\
$A_{B},(\mathrm{mag})$ & 0.22 & 0.07 & 0.07 & 0.01 & 0.00 & 0.00 \\
Type & $\mathrm{SBdm}$ & $\mathrm{SA}(\mathrm{rs}) \mathrm{b}$ & $\mathrm{SAB}(\mathrm{rs}) \mathrm{bc}$ & $\mathrm{SA}(\mathrm{s}) \mathrm{m}$ & $\mathrm{SA}(\mathrm{s}) \mathrm{cd}$ & $\mathrm{SAB}(\mathrm{s}) \mathrm{d}$ \\
$V_{\mathrm{h}},\left(\mathrm{km} \mathrm{s}^{-1}\right)$ & 198 & 411 & 556 & 201 & 273 & 305 \\
$V_{0},\left(\mathrm{~km} \mathrm{~s}^{-1}\right)$ & 389 & 364 & 443 & 341 & 412 & 459 \\
$N_{*} *$ & 84 & 327 & 273 & 131 & 298 & 102 \\
$\langle B(3 B)\rangle,(\mathrm{mag})$ & 20.39 & 20.64 & 20.27 & 19.81 & 20.37 & 20.71 \\
$\langle B-V\rangle_{3}$ & -0.17 & 0.10 & 0.14 & 0.14 & 0.15 & 0.08 \\
$(m-M)_{0}$ & 28.47 & 29.81 & 29.74 & 28.06 & 29.15 & 29.70 \\
$D,(\mathrm{Mpc})$ & 5.0 & 9.2 & 8.9 & 4.1 & 6.8 & 8.7 \\
$M_{B}$ & -16.46 & -19.24 & -20.13 & -16.34 & -18.22 & -18.50 \\
$H=V_{0} / D$ & 78 & 40 & 50 & 83 & 61 & 53 \\
$\mathrm{SGB},\left(^{\circ}\right)$ & -6.3 & -33.4 & -36.4 & 17.8 & 22.9 & 24.7 \\
\hline
\end{tabular}

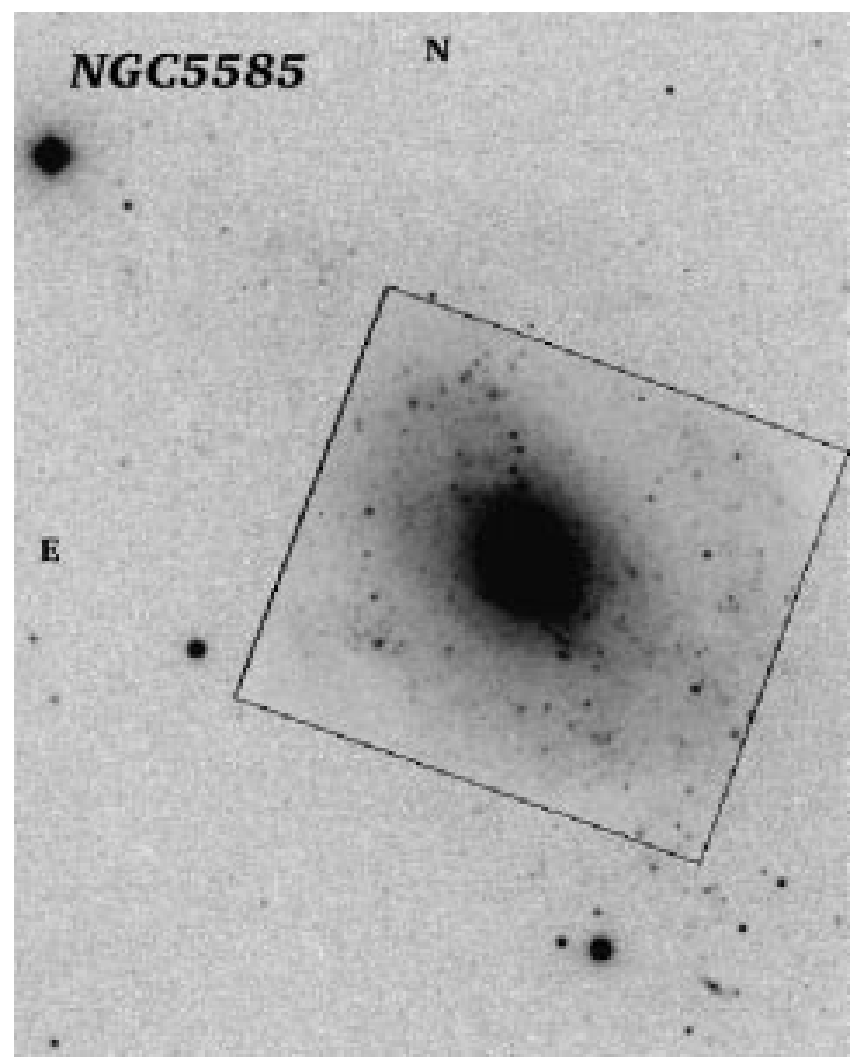

Fig. 14. $I$ band inlay of NGC 5585 from the $1 \mathrm{~m}$ Kapteyn telescope image with the position of the $6 \mathrm{~m}$ BTA CCD frame

increasing galaxy supergalactic latitude, SGB (the last line in Table 2). This tendency agrees well with the general picture of an anisotropic expansion of the Local volume, which seems slower in the directions of the poles of the Local "pancake" (Karachentsev \& Makarov 1996).

Acknowledgements. This work was supported by INTASRFBR through grant 95-IN-RU-1390. We have made use of the Nasa/IPAC Extragalactic Database (NED, which is operated by the Jet Propulsion Laboratory, Caltech, under contract with the National Aeronautics and Space Administration), Royal Greenwich Observatory/Isaac Newton Group Archives, and the Digitized Sky Survey (DSS-1) produced at the Space Telescope Science Institute under U.S. Government grant NAG W-2166.

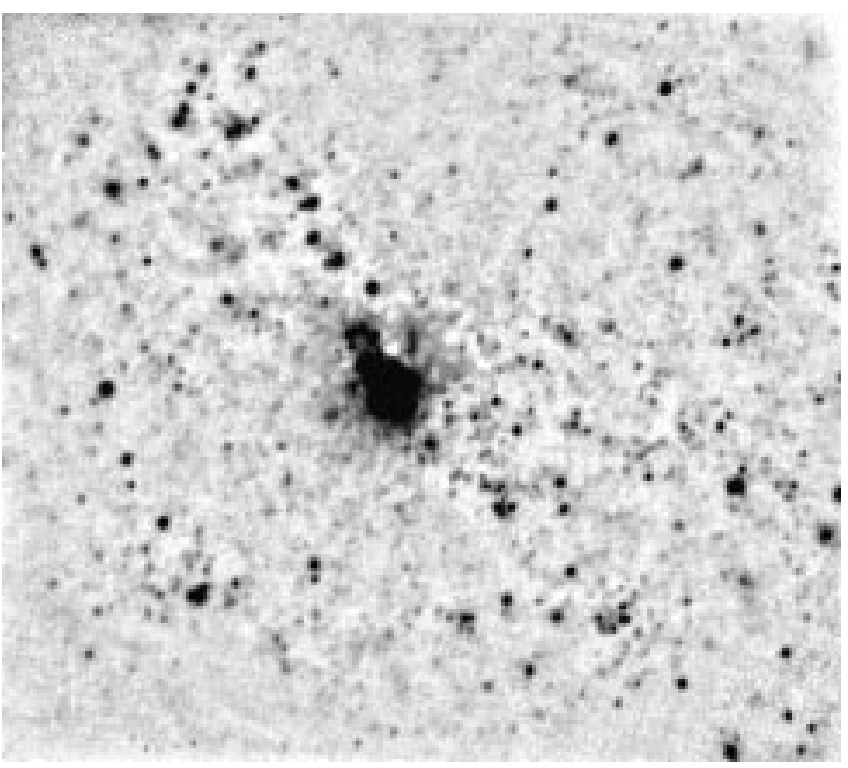

Fig. 15. The 6-m telescope $I$ frame of NGC 5585 after subtracting median, smoothed with a window of $10 \times(F W H M)$

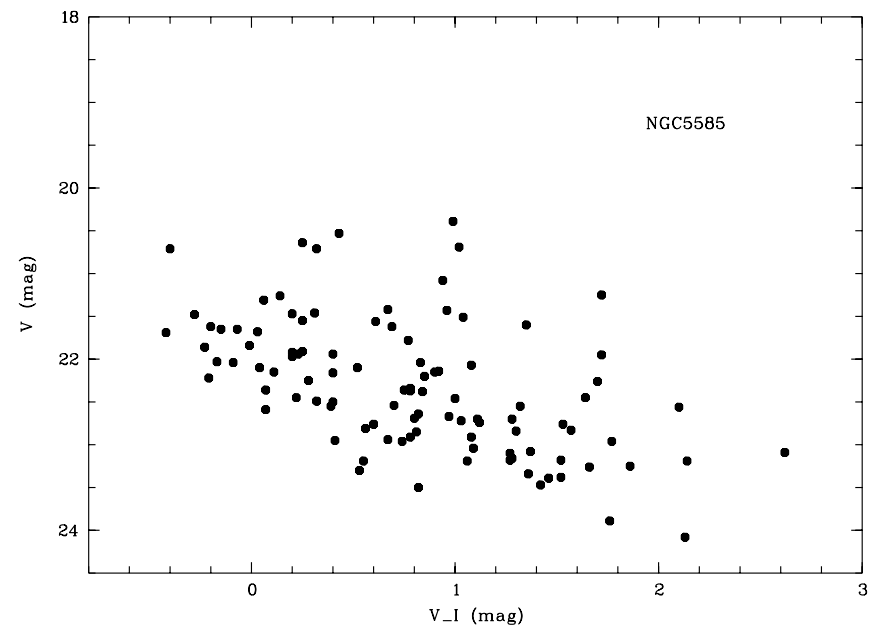

Fig. 16. $V$ vs. $(V-I)$ CM diagram for 102 stars from the two regions studied in NGC 5585 


\section{References}

Ables D.R., Cook K.H., 1995, AJ 100, 192

Broelis A.H., van Woerden H., 1994, A\&AS 107, 129

de Vaucouleurs G., 1978, ApJ 224, 14

Georgiev Ts.B., Karachentsev I.D., Tikhonov N.A., 1997, Lett. Astron. Zh. 23, 586

Huchtmeier W.H., Karachentsev I.D., Karachentseva V.E., Ehle M., 2000, A\&AS (submitted)

Karachentseva V.E., 1973, Communic. Spec. Astrophys. Obs. 8,3

Karachentsev I., 1994, A\&A Trans. 6, 1

Karachentsev I., Drozdovsky I., 1998, A\&AS 131, 1

Karachentsev I., Drozdovsky I., Kajsin S., et al., 1997, A\&AS 124,559

Karachentsev I.D., Karachentseva V.E., 1998, A\&AS 127, 409

Karachentsev I.D., Kopylov A.I., Kopylova F.G., 1994, Bull.
Spets. Astrofiz. Obs. 38, 1

Karachentsev I.D., Makarov D.I., 1996, AJ 111, 535

Karachentsev I.D., Tikhonov N.A., 1994, A\&A 286, 718

Kraan-Korteweg R., Tammann G., 1979, Astron. Nachr. 300, 181

Landolt A.U., 1992, AJ 104, 340

Makarova L.N., Karachentsev I.D., 1998, A\&AS 133, 181

Makarova L.N., Karachentsev I.D., Georgiev Ts.B., 1997, Lett. Astron. Zh. 23, 435

Sandage A., Bedke J., 1988, Atlas of galaxies useful for measuring the cosmological distance scale, NASA, Washington, 1 Sandage A.R., Tammann G.A., 1974, ApJ 190, 525; 194, 223 Stetson P., 1987, PASP 99, 191

Tully R.B., 1988, Nearby Galaxy Catalog. Cambridge Univ. Press, Cambridge

Tully R.B., Fisher J.R., 1977, A\&A 54, 611 